\title{
The Cystic Fibrosis Transmembrane Conductance Regulator 470 Met Allele Is Associated with an Increased Risk of Chronic Pancreatitis in Both Asian and Caucasian Populations: A Meta-Analysis
}

\author{
Donger Zhou, ${ }^{1}$ Rui Bai, ${ }^{2}$ and Liang Wang ${ }^{1}$
}

\begin{abstract}
Background: The Met470Val polymorphism (1540A $>\mathrm{G}$ [rs213950]) within the cystic fibrosis transmembrane conductance regulator (CFTR) protein has been reported to be associated with chronic pancreatitis (CP). The results remain inconclusive, and therefore, we performed this meta-analysis to clarify the association between M470V and CP risk.

Methodology/Results: We conducted a meta-analysis of 7 case-control studies, including a total of 1121 CP patients and 2209 controls from Asian and Caucasian populations. We calculated the odds ratio (OR) and 95\% confidence intervals (95\% CI). Met $470 \mathrm{Val}$ was found to be significantly associated with an increased risk of $\mathrm{CP}$ under all the genetic models ( $\mathrm{M}$ vs. $\mathrm{V}, \mathrm{OR}=1.260,95 \% \mathrm{CI}: 1.134-1.399$; MV vs. VV, OR $=1.292,95 \%$ CI: $1.091-1.530 ;$ MM vs. VV, OR $=1.579,95 \%$ CI: $1.274-1.956$; MV/MV vs. VV, OR $=1.366,95 \%$ CI: $1.165-$ 1.603; $\mathrm{MM}$ vs. $\mathrm{MV} / \mathrm{VV}, \mathrm{OR}=1.346,95 \% \mathrm{CI}$ : $1.114-1.621$ ). Met470Val was also found to be significantly associated with an increased risk of idiopathic CP (ICP) in allele contrast, codominant, and recessive models ( $\mathrm{M}$ vs. $\mathrm{V}, \mathrm{OR}=1.298,95 \% \mathrm{CI}: 1.020-1.653$; $\mathrm{MV}$ vs. $\mathrm{VV}, \mathrm{OR}=1.297,95 \% \mathrm{CI}: 1.074-1.566$; $\mathrm{MM}$ vs. VV, $\mathrm{OR}=1.473,95 \% \mathrm{CI}: 1.165-1.862 ; \mathrm{MM}$ vs. $\mathrm{MV} / \mathrm{VV}, \mathrm{OR}=1.254,95 \% \mathrm{CI}: 1.023-1.538)$.

Conclusions: The CFTR $470 \mathrm{M}$ allele is significantly associated with an increased risk of CP in both Asian and Caucasian populations. The CFTR $470 \mathrm{M}$ allele is also significantly associated with risk of ICP.
\end{abstract}

Keywords: CFTR, polymorphism, chronic pancreatitis, meta-analysis

\section{Introduction}

C HRONIC PANCREATITIS (CP) is a chronic inflammatory syndrome of the pancreas, characterized by recurrent abdominal pain; fibrosis and atrophy of the pancreatic parenchyma; and loss of exocrine and endocrine tissue (Witt et al., 2007; Issa et al., 2014). The etiologic factors underlying $\mathrm{CP}$, including alcohol toxicity, anatomic abnormalities, autoimmunity, and genetic predisposition, interact with each other leading to a complex pathogenesis. $\mathrm{CP}$ is frequently subclassified according to epidemiologic factors including but not limited alcoholism, familial history, and autoimmune disorders. When no specific factor can be identified, it is classified as idiopathic chronic pancreatitis (ICP).

Although the pathogenesis of $\mathrm{CP}$ remains unclear, certain genetic factors have been shown to contribute to $\mathrm{CP}$ progression (Hanck et al., 2003; Thrower et al., 2008). Multiple studies have suggested a link between the cystic fibrosis transmembrane conductance regulator $(C F T R)$ gene and $\mathrm{CP}$ (Audrezet et al., 2002; Keiles and Kammesheidt, 2006). The CFTR gene is positioned on chromosome 7q31 and contains 27 exons, encoding a cAMP regulated $\mathrm{Cl} 2$-channel protein expressed on the epithelial cell membrane and regulating other membrane transport proteins (Moskowitz et al., 2008; Tomaiuolo et al., 2015). More than 2000 mutations and polymorphic loci have been characterized in the CFTR gene (Cystic Fibrosis Mutation Database). Among them is an amino acid changing polymorphism (Met470Val; 1540A $>\mathrm{G}$ [rs213950]) in exon 10, which has been associated with CP (Lee et al., 2003; Fujiki et al., 2004).

The M470V polymorphism is often investigated with other polymorphisms such as the 5-thymidine (5T) repeats and the TG repeats. Haplotypes defined by various combinations of $\mathrm{M} 470 \mathrm{~V}, 5 \mathrm{~T}$, and TG repeats were found to be associated with

${ }^{1}$ Department of Hepatobiliary-Pancreatic Surgery and ${ }^{2}$ Cancer Institute (Key Laboratory of Cancer Prevention and Intervention, China National Ministry of Education), The Second Affiliated Hospital, Zhejiang University School of Medicine, Zhejiang, Hangzhou, China. 
$\mathrm{CP}$; the results, however, are not definitive. Chang et al. (2007) reported that M470V alone was not associated with $\mathrm{CP}$, but the haplotype of $125 \mathrm{G} / 1001+11 \mathrm{C} / \mathrm{TG} 12 / \mathrm{M} 470 /$ $2694 \mathrm{~T} / 4521 \mathrm{G}$ was associated with the risk of CP; Lee et al. (2003) reported that V470 with the Q1352H mutation was strongly associated with the risk of CP; and Steiner et al. (2011) reported that the haplotype of TG10-T7-M470 was associated with the risk of $\mathrm{CP}$. The multiple haplotypes containing the M470V polymorphism examined in these studies make the analysis of this single marker's role difficult to assess in the pathogenesis of CP.

We therefore performed a systematic review of the literature and conducted a meta-analysis focused only on the M470V polymorphism to estimate its involvement in CP risk.

\section{Methods}

\section{Identification of eligible studies}

We carried out a systematic search of the PubMed and Embase databases through August 15, 2019. The following terms were used: "CF transmembrane conductance regulator," "rs213950," “M470V," "Single Nucleotide Polymorphism" and "Chronic pancreatitis," without any other limitation applied. The reference lists of the retrieved studies and reviews were manually searched for further relevant studies. Duplicate publications and irrelevant articles were excluded.

\section{Inclusion and exclusion criteria}

Studies included for the meta-analysis should conform to the following criteria: (1) case-control studies; (2) investigating the association between p.Met470Val (rs213950) and $\mathrm{CP}$; (3) $\mathrm{CP}$ diagnoses via standard guidelines or diagnostic criteria; and (4) providing detailed genotypic frequencies of rs213950. Studies without detailed genotypic frequencies were excluded. Titles and abstracts of the search results were screened, followed by a full-text review of each article. Two reviewers (D.Z and R.B.) extracted all eligible studies independently and the disagreements were resolved by discussion.

\section{Data extraction}

Data from the included studies were extracted by two reviewers (D.Z. and R.B.) independently. The following data were collected: name of first author, year of publication, country where the study was conducted, genotyping methods, ethnicity, and etiology of CP, number of cases and controls, genotype frequency in cases and controls. Different ethnicities were categorized as Asian or Caucasian. Data regarding ICP were also collected. Hardy-Weinberg equilibrium (HWE) in the controls was calculated and $p<0.05$ was considered a significant departure from HWE. Two reviewers reached a consensus on each item and disagreements were solved via discussion with another reviewer (L.W.).

\section{Statistical analysis}

We conducted and reported our meta-analysis in accordance with the guideline of the Meta-analysis of Observational Studies in Epidemiology (MOOSE) Group (Stroup et al., 2000). The association strength between p.V470M (rs213950) and CP was measured by odds ratio (OR) with 95\% confidence intervals $(95 \% \mathrm{CI}$ ) (Lau et al., 1997). The pooled ORs were calculated for allele contrast (M vs. V), codominant model (MM vs. VV, MV vs. VV), dominant (MM MV vs. VV), and recessive (MM vs. MV VV) models, assuming dominant and recessive effects of the variant $\mathrm{M}$ allele, respectively. Subgroup analyses were also applied based on ethnicities. Sensitivity analyses were performed to identify an individual study's effect on the pooled results. The chi-square based $Q$-test was used to check the statistical heterogeneity among studies, and heterogeneity was considered statistically significant when $p<0.10$. The fixed-effects model (based on Mantel-Haenszel method) was used when there was no significant heterogeneity; otherwise, the random-effects model (based on DerSimonian-Laird method) was applied (DerSimonian and Laird, 2015). Publication bias was detected using Begg's funnel plot and the Egger' linear regression test, and a $p<0.05$ was considered statistically significant (Egger et al., 1997). All statistical analyses were calculated with STATA software (version 14.0; StataCorp, College Station, TX). Also, all $p$-values were two-sided.

\section{Results \\ Characteristics of eligible studies}

A total of six articles (Lee et al., 2003; Fujiki et al., 2004; Chang et al., 2007; Tzetis et al., 2007; de Cid et al., 2010; Steiner et al., 2011) were included based on the predetermined inclusion and exclusion criteria. The detailed screening process is shown in Figure 1. In the article by Steiner et al. (2011), there were two separate studies, one was carried out in Switzerland consisting of persons from the Czech-Republic, France, Italy, and Switzerland, and the other study was from Germany and comprised only Germans, with these studies using a different genotyping methods. Thus, a total of seven case-control studies were included in this meta-analysis (shown in Table 1), which include a combined 1121 patients suffering from CP and 2209 controls. Four of the studies were carried out among Caucasians and three among Asians. The different genotyping methods used in each study and the presumed etiology of CP are shown in Table 1. In all the studies, the HWE of genotype distribution of the control group were calculated and found to be consistent with HWE.

\section{Meta-analysis results}

The results showed that M470 had a significant association with increased risk of CP under all models of inheritance evaluated, including the allele contrast ( $\mathrm{M}$ vs. V, $\left.\mathrm{OR}=1.260,95 \% \mathrm{CI}: 1.134-1.399, p_{\text {heterogeneity }}=0.303\right)$; the codominant model ( $\mathrm{MV}$ vs. $\mathrm{VV}, \mathrm{OR}=1.292,95 \% \mathrm{CI}$ : $1.091-1.530, p_{\text {heterogeneity }}=0.153 ; \mathrm{MM}$ vs. VV, OR $=1.579$, 95\% CI: $\left.1.274-1.956, p_{\text {heterogeneity }}=0.273\right)$; the dominant model (MV/MV vs. VV, OR $=1.366,95 \% \mathrm{CI}: 1.165-1.603$, $\left.p_{\text {heterogeneity }}=0.213\right)$; and the recessive $\operatorname{model}(\mathrm{MM}$ vs. $\mathrm{MV} / \mathrm{VV}$, $\left.\mathrm{OR}=1.346,95 \% \mathrm{CI}: 1.114-1.621, p_{\text {heterogeneity }}=0.219\right)$. The results are shown in Table 2 and Figures 2 and 3.

In the subgroup analysis, ethnicity was added to the investigation. Among the Asian studies, a significant association between M470 and CP was observed in the allele contrast (M vs. V, OR $=1.295,95 \%$ CI: $\left.1.009-1.662, p_{\text {heterogeneity }}=0.895\right)$ and codominant models ( $\mathrm{MM}$ vs. $\mathrm{VV}, \mathrm{OR}=1.666,95 \% \mathrm{CI}$ : $\left.1.022-2.715, p_{\text {heterogeneity }}=0.937\right)$. In the Caucasian group, 


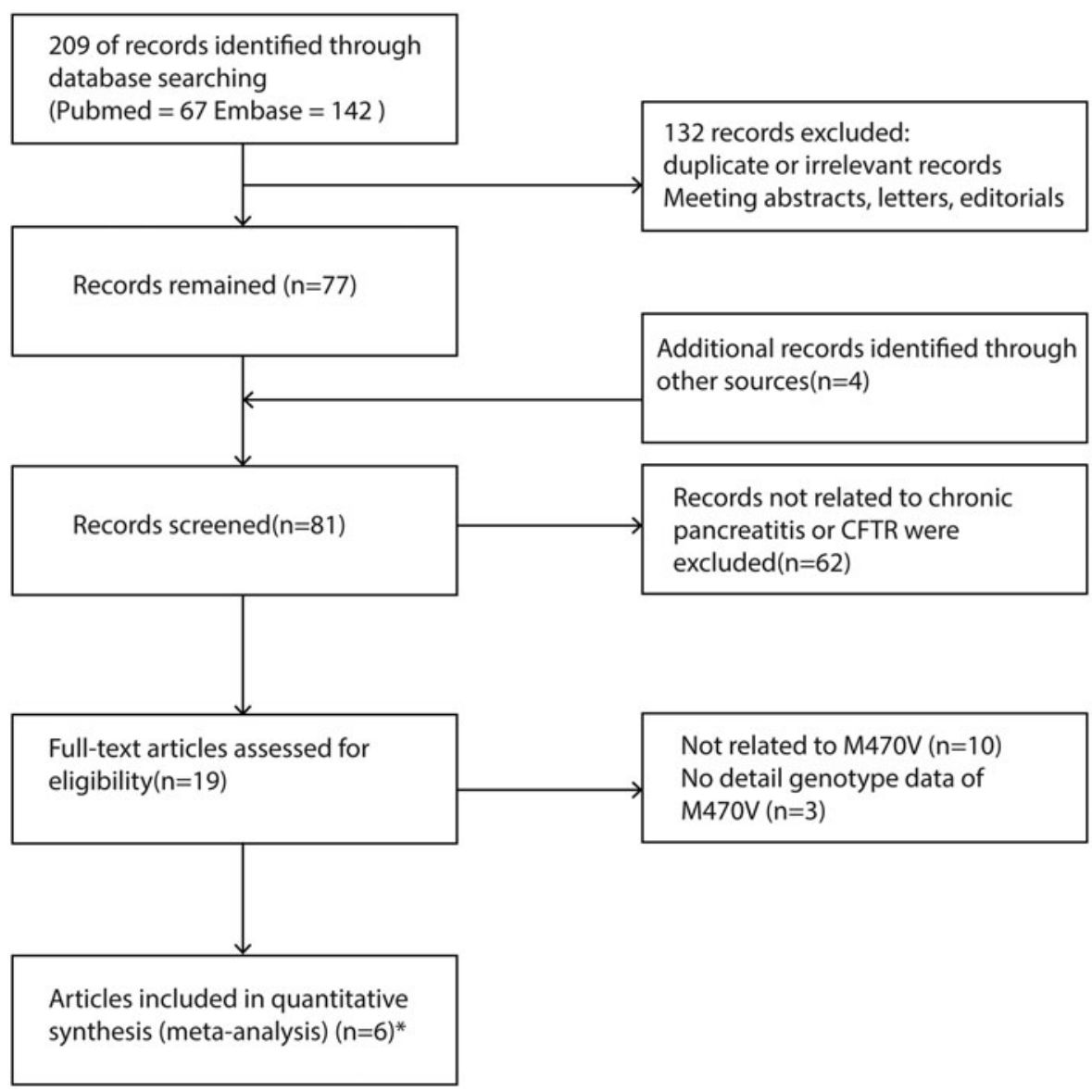

significant heterogeneity was observed; therefore, we applied a random-effects model when $p_{\text {heterogeneity }}<0.1$. A significant association was also observed in the Caucasian group under the allele contrast model ( $\mathrm{M}$ vs. $\mathrm{V}, \mathrm{OR}=1.392,95 \% \mathrm{CI}$ : $\left.1.098-1.765, p_{\text {heterogeneity }}=0.075\right)$; the codominant model (MM vs. VV, OR $=2.011,95 \%$ CI: $1.083-2.268, p_{\text {heterogeneity }}=$ 0.084 ); and the dominant model (MV/MV vs. VV, OR $=1.567$, 95\% CI: $\left.1.083-2.268, p_{\text {heterogeneity }}=0.084\right)$. Results are shown in Table 2.

We also performed an analysis of the ICP cases, that is, no etiology reported; and we observed significant heterogeneity. Therefore, we applied the random-effects model when $p_{\text {het- }}$ erogeneity $<0.1$ and found that a strong association existed between M470 and ICP under the allele contrast model (M vs. $\mathrm{V}, \mathrm{OR}=1.298,95 \% \mathrm{CI}: 1.020-1.653, p_{\text {heterogeneity }}=$ 0.082 ); the codominant model ( $\mathrm{MV}$ vs. $\mathrm{VV}, \mathrm{OR}=1.297$, $95 \%$ CI: $1.074-1.566, p_{\text {heterogeneity }}=0.109 ; \mathrm{MM}$ vs. VV, OR = 1.473, 95\% CI: $1.165-1.862, p_{\text {heterogeneity }}=0.143$ ); and the recessive model (MM vs. $\mathrm{MV} / \mathrm{VV}, \mathrm{OR}=1.254,95 \% \mathrm{CI}$ : $1.023-1.538, p_{\text {heterogeneity }}=0.378$ ). The results are shown in Table 2 and Figures 4 and 5.

\section{Sensitivity analysis}

A sensitivity analysis was performed using the method of deleting a single study each time and recalculating the pooled OR to explore the excluded study's influence on the final result. We performed the analysis of overall comparison for all of the inheritance models. As shown in Figure 6, after deleting the study by Steiner group 2, the recalculated OR was more than the upper CI limit $(95 \% \mathrm{CI})$ of the original one in allele contrast. But the same trend could be drawn and the risk was even more significant after deleting the last study. Similar situations were seen in other model comparison (data not shown). So the results are robust.

\section{Publication bias}

Publication bias was assessed by Begg's funnel plot and Egger's test. No evidence of publication bias was observed (Fig. 7).

\section{Discussion}

The M470V polymorphism is a missense single nucleotide variant located in exon 10 of the CFTR gene, in which the M allele is the ancestral allele. However, while the $M$ allele is the major allele in African populations, it has been reported to be the minor allele in non-African populations, including the European and Asian populations (Kosova et al., 2010) according to the International HapMap Project (International HapMap, 2005) and the Human Genome Diversity Project (HGDP) (Cann et al., 2002). The frequencies of the $\mathrm{V}$ allele were 0.93 in Tuscans and 0.80 in Mongolians in the database (Kosova et al., 2010). In our study, the combined frequency of the $\mathrm{V}$ allele in the control group is 0.59 in total $(0.60$ in Asian group and 0.59 in European group), which is consistent with previous reports (Chang et al., 2007; Rosendahl et al., 2013). Kosova et al. (2010) postulated that Val470 might provide a positive selection because the $M$ allele was 

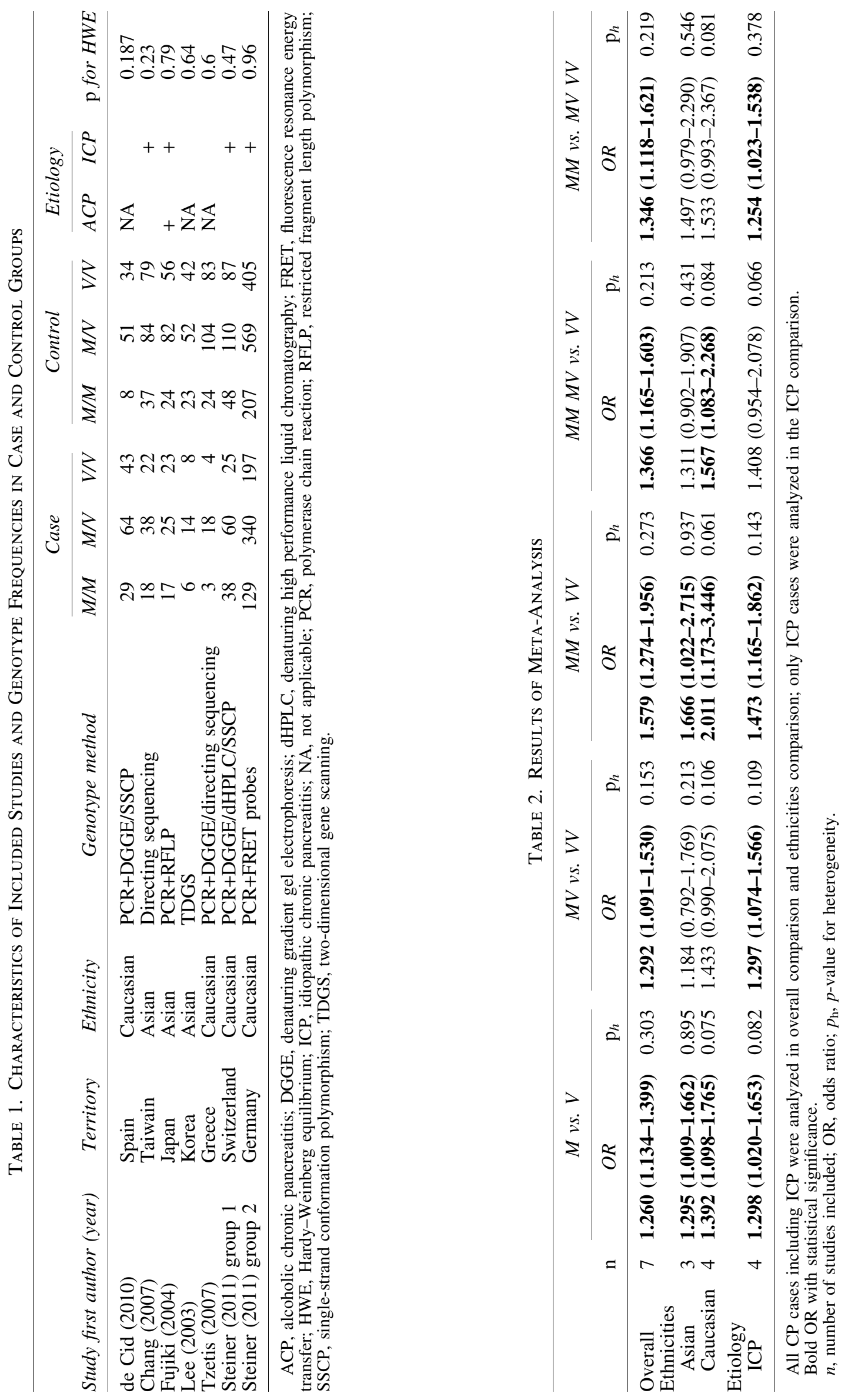
OR $(95 \% \mathrm{Cl})$

Weight

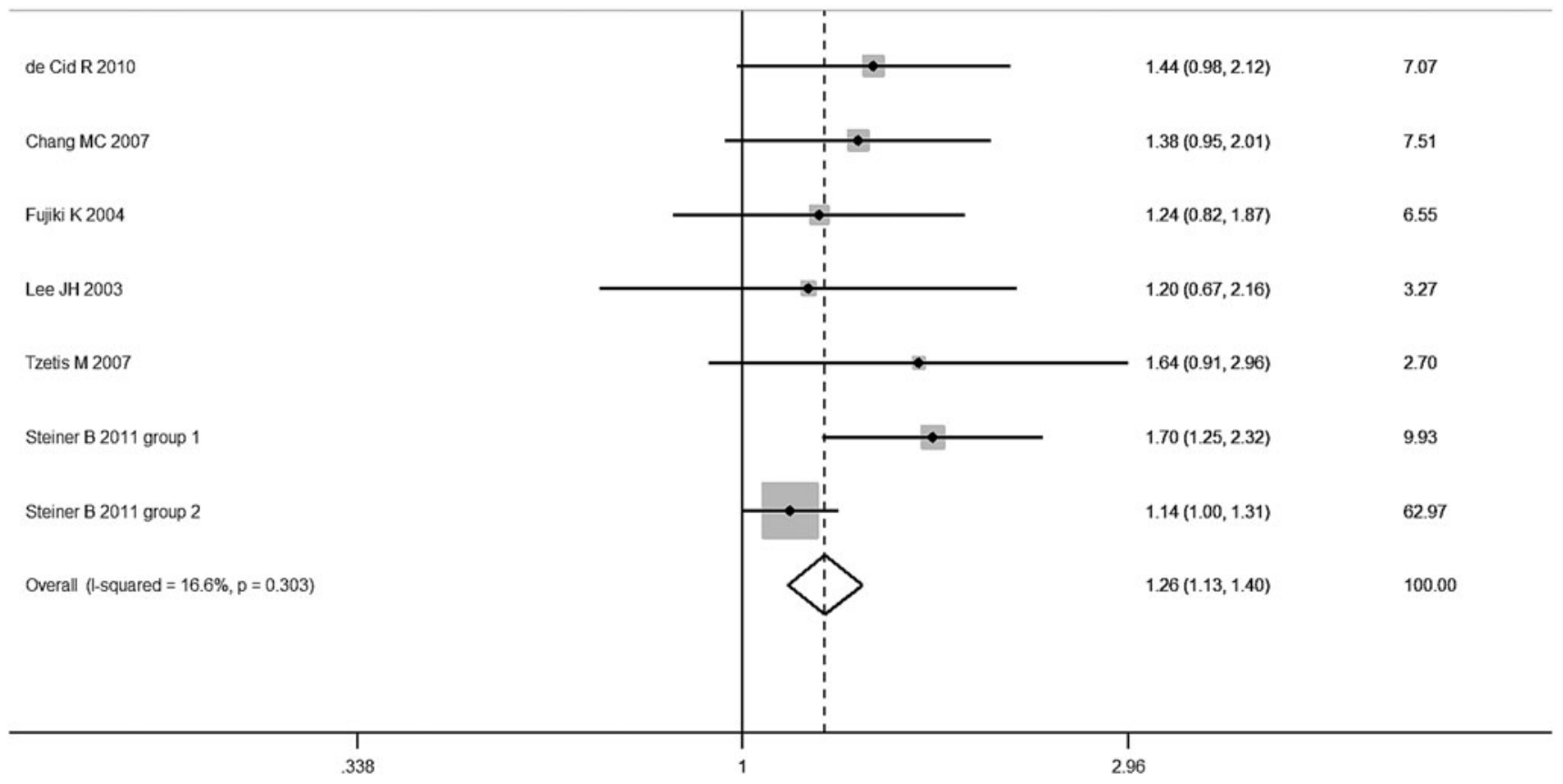

FIG. 2. Forest plot of allele contrast for overall comparison ( $\mathrm{M}$ vs. V) using a fixed-effect model. All CP cases including ICP were analyzed in overall comparison. ICP, idiopathic chronic pancreatitis.

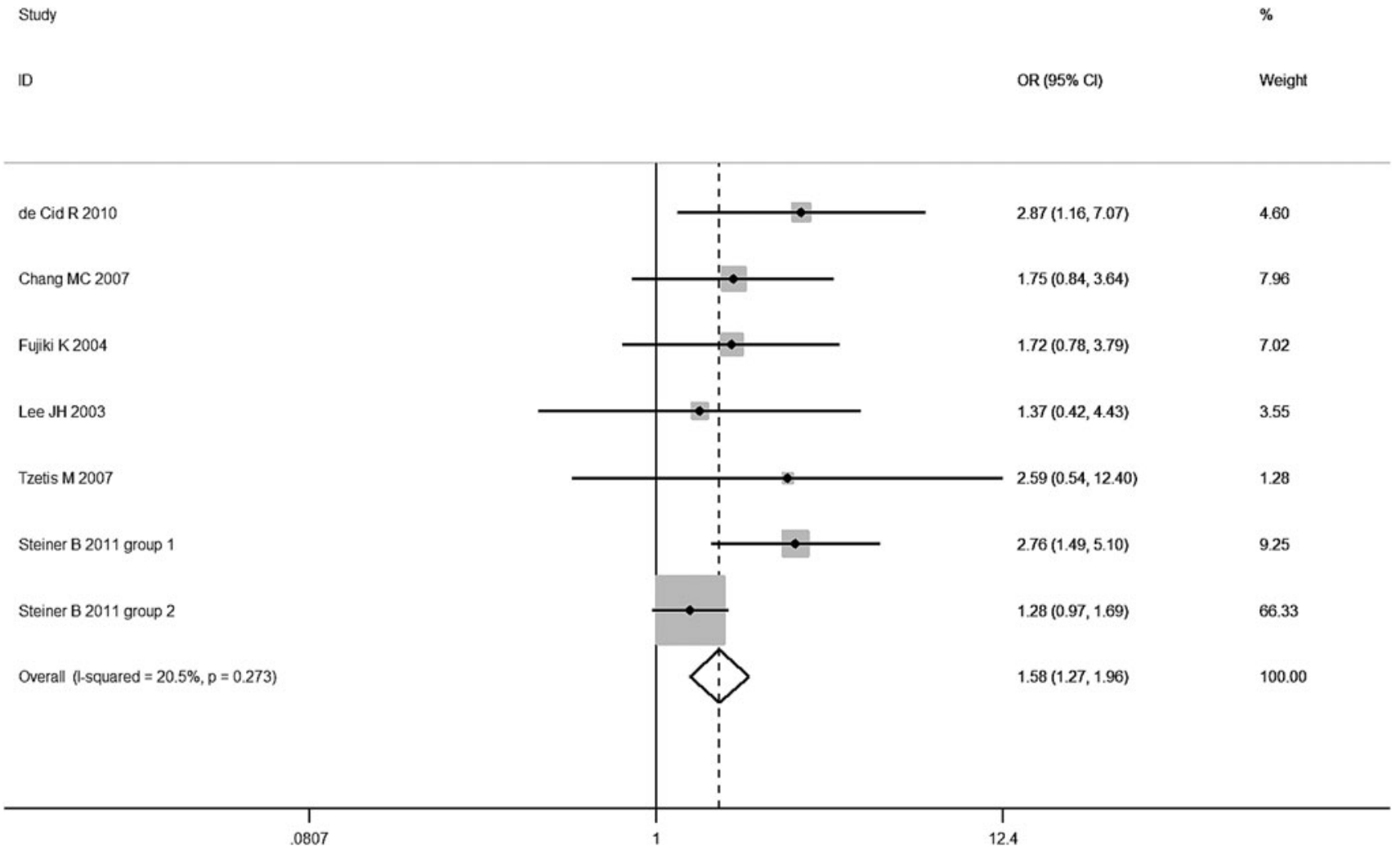

FIG. 3. Forest plot of homozygote comparison for overall comparison (MM vs. VV) using a fixed-effect model. All CP cases including ICP were analyzed in overall comparison. 


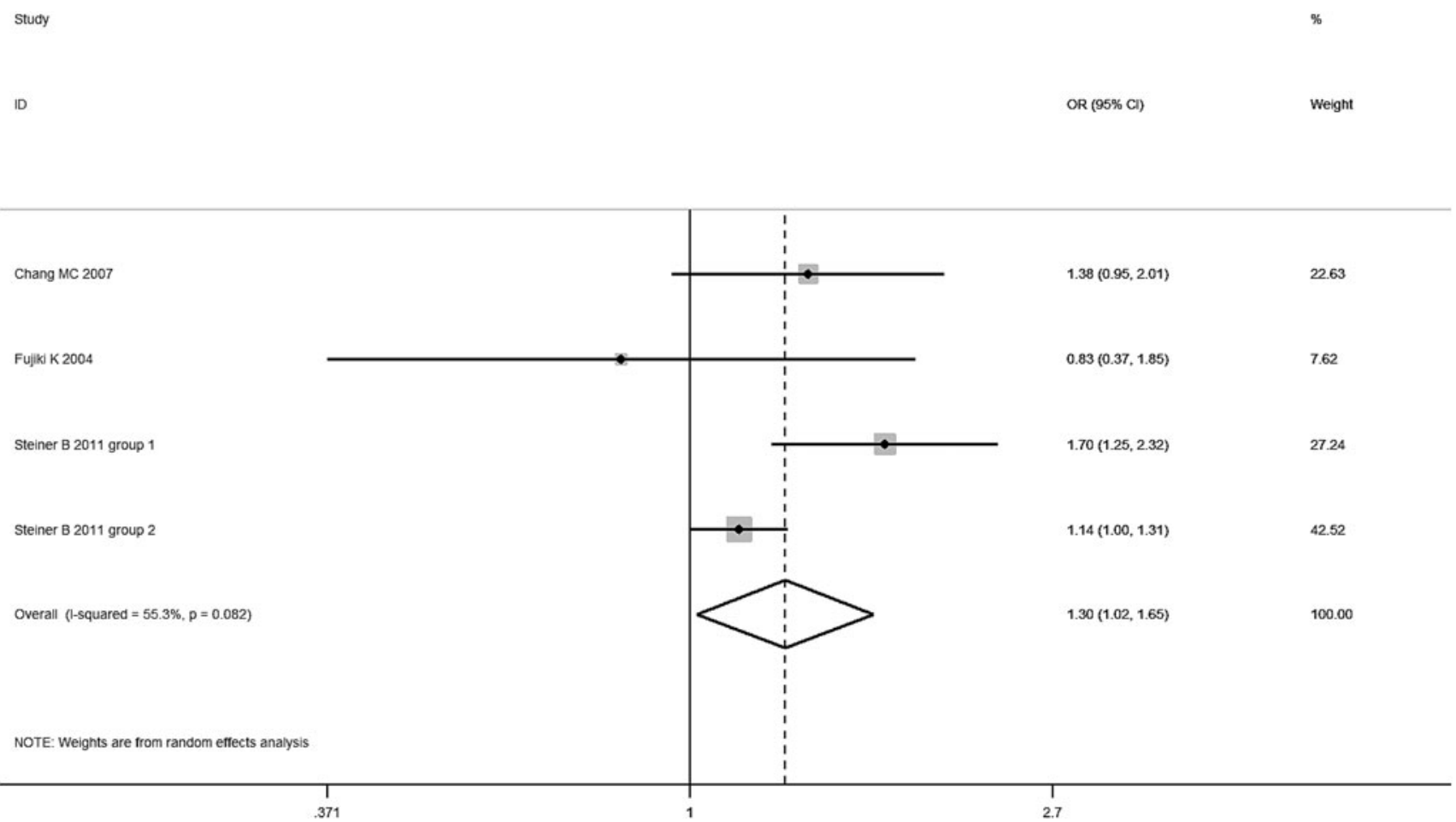

FIG. 4. Forest plot of allele contrast for ICP (M vs. V) using a random-effects model. Only ICP cases were analyzed in this comparison.

associated with disadvantageous fertility effects. In addition, there have been several studies examining the relationship between the M470 allele and other diseases. Ciminelli et al. (2007) reported that the $\mathrm{M}$ allele was highly associated with CF-causing mutations in CF. Steiner et al. (2011) reported

Study

ID

Fujiki K 2004

Steiner B 2011 group 1

Steiner B 2011 group 2

Overall (1-squared $=44.7 \%, p=0.143$ )

NOTE: Weights are from random effects analysis that the TG10-T7-M470 haplotype increased the risk of ICP and congenital bilateral absence of the vas deferens (CBAVD). Our results showed that M470 independently increases the risk of $\mathrm{CP}$, including ICP, in both Asian and European populations.

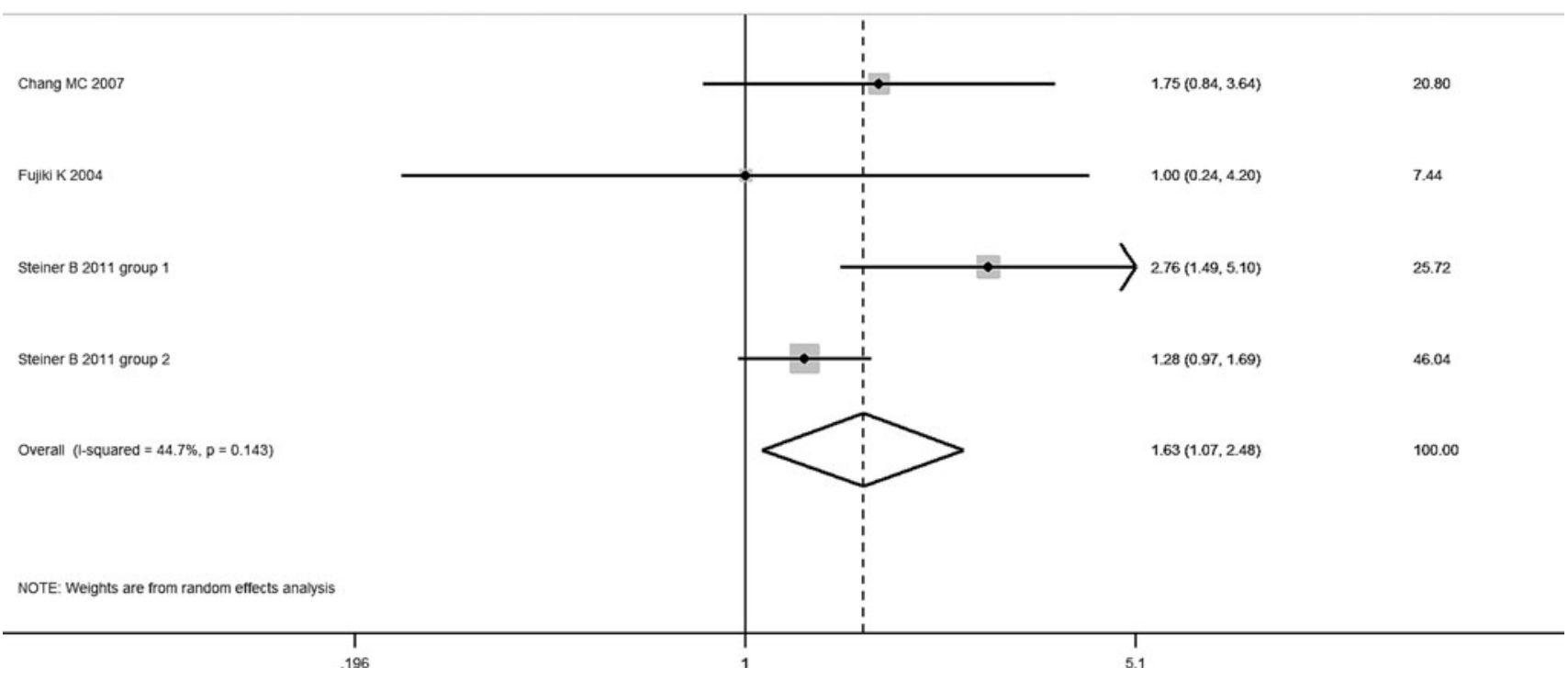

FIG. 5. Forest plot of homozygote comparison for ICP (MM vs. VV) using a random-effects model. Only ICP cases were analyzed in this comparison. 
FIG. 6. Sensitivity test of allele contrast for overall comparison ( $\mathrm{M}$ vs. $\mathrm{V}$ ).

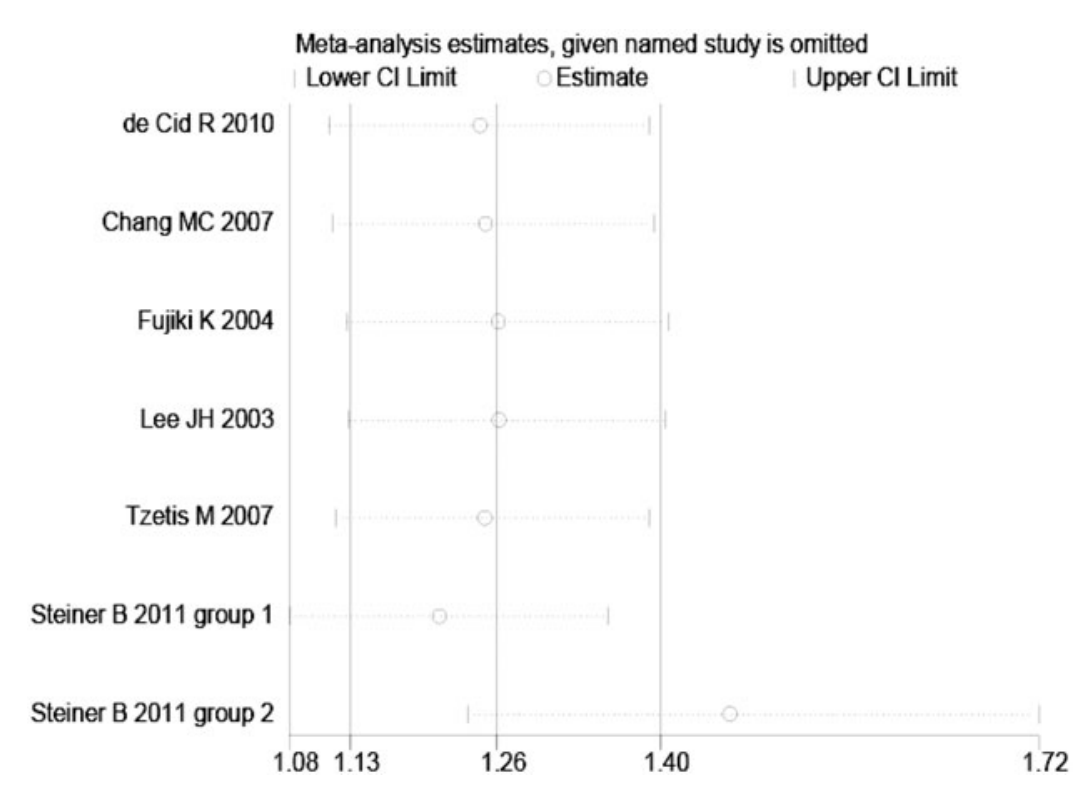

Little is understood about the pathophysiological role of M470V in the CFTR protein in CP. Val470 causes the CFTR protein to mature quickly but with a lower activity, while Met470 results in an intact protein with normal activity that matures slowly (Cuppens et al., 1998). Several studies have shown that M470V may act like a modifier by increasing the penetrance of other mutations or polymorphisms (de Meeus et al., 1998). For example, Lee et al. (2003) reported that E217G and Q1352H on the M470 background can both decrease membrane protein expression and anion transporting activities by $60-70 \%$, and that Q1352H in the V470 background can also arise in the low activity type of V470, so that either $\mathrm{M}$ or $\mathrm{V}$ can combine with other polymorphisms to confer a dysfunction of the CFTR protein. However, it has been reported (Pompei et al., 2006; Ciminelli et al., 2007) that the majority of the disease-causing muta- tions, including but not limited to F508del, N1303K, and W1282X, occur in the M470 background, and that this may be one of the reasons that M470 was associated with an increased risk of CP. There was also conjecture (Steiner et al., 2011) that the slower maturation of the $M$ allele may not affect the intrinsic chloride channel activity, but may impair the tissue- and development-specific maturation and turnover of a functional CFTR channel. As we do not know the function of the CFTR clearly, additional mechanistic work needs to be done to functionally characterize the physiological role of the various CFTR protein polymorphisms and their contribution to in CP.

There are some advantages to this meta-analysis. This is the first meta-analysis to focus solely on the association between M470V and CP. Previous meta-analyses concerning the CFTR gene and CP usually focused on known deleterious
FIG. 7. Begg's funnel plot of allele contrast for overall comparison (M vs. V).

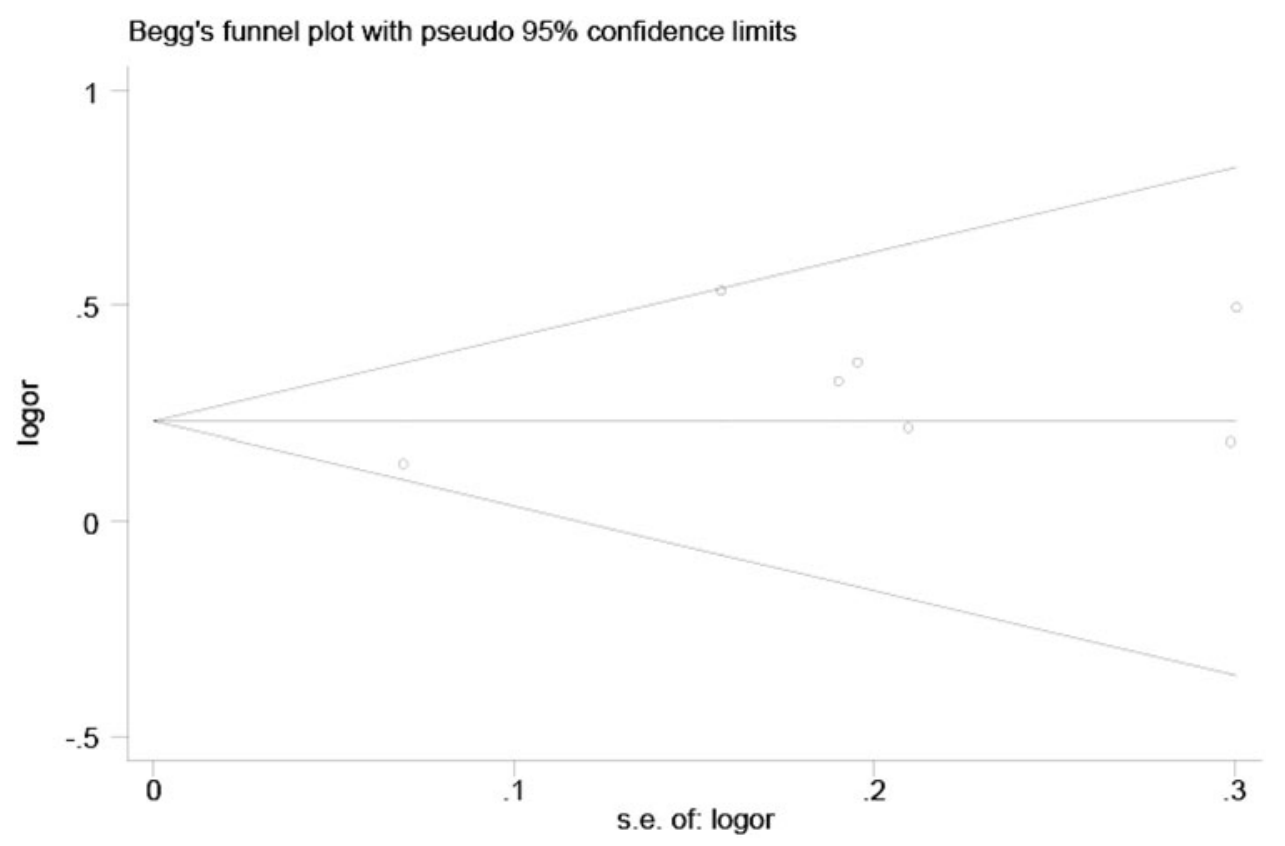


mutations and not structural polymorphisms that were not predicted to interfere with protein function. In this study, we demonstrated for the first time that het M470V polymorphism in the CFTR gene was independently associated with the risk of CP. In addition, there were no limitations in the literature search, and thus selection bias was well controlled. There was also no publication bias identified by either Begg's funnel plot or Egger's regression test.

This meta-analysis was not without its limitations. First, in the subgroup analysis, the number of studies in each subgroup was limited and the sample size among the studies was significant. These may contribute to heterogeneity in some genetic models. Second, 932 (83\%) of $1121 \mathrm{CP}$ patients were ICP patients, and therefore ICP contributes to the majority of the patient group. ICP was defined as $\mathrm{CP}$ without obvious epidemiologic factors, which might lead the ICP more likely to be caused by genetic predisposition. We should be cautious when interpreting the relationship between M470 and CP with mixed etiology. More samples of CP with mixed etiology should be included in a future study. Third, the ethnicities in this study were Asian and Caucasian and these populations have a similar genetic background where the $\mathrm{M}$ allele is the minor allele. The $\mathrm{M}$ allele, however, is the major allele in African populations; there have been no studies on the CFTR genes effects on $\mathrm{CP}$ among this population to our knowledge. It would be interesting to also investigate the role of $\mathrm{M} 470 \mathrm{~V}$ in $\mathrm{CP}$ in other ethnic groups.

In summary, we analyzed seven case-control studies, which included $1121 \mathrm{CP}$ patients and 2209 controls for this meta-analysis. We demonstrated that the CFTR $470 \mathrm{M}$ allele independently is strongly associated with an increased risk of $\mathrm{CP}$ both in Asian and Caucasian populations. The same significant association was also observed in ICP patients.

\section{Authors' Contributions}

D.Z. and R.B. confirmed the topic of this article. D.Z., R.B., and L.W. performed the statistical analysis and analyzed the results. R.B. and L.W. contributed to revising the article. D.Z. wrote the article. All authors approved the final version of the article.

\section{Author Disclosure Statement}

No competing financial interests exist.

\section{Funding Information}

The present study was supported by grants from the Natural Science Foundation of Zhejiang Province, China (LY17H160035 and LQ15H160010), the National Science Foundation of China (No. 81702382), and Zhejiang Basic Public Welfare Research Project (LGF18H160040 to R.B.)

\section{References}

Audrezet MP, Chen JM, Le Marechal C, et al. (2002) Determination of the relative contribution of three genes-the cystic fibrosis transmembrane conductance regulator gene, the cationic trypsinogen gene, and the pancreatic secretory trypsin inhibitor gene-to the etiology of idiopathic chronic pancreatitis. Eur J Hum Genet 10:100-106.
Cann HM, de Toma C, Cazes L, et al. (2002) A human genome diversity cell line panel. Science 296:261-262.

Chang MC, Chang YT, Wei SC, et al. (2007) Spectrum of mutations and variants/haplotypes of CFTR and genotypephenotype correlation in idiopathic chronic pancreatitis and controls in Chinese by complete analysis. Clin Genet 71:530 539.

Ciminelli BM, Bonizzato A, Bombieri C, et al. (2007) Highly preferential association of NonF508del CF mutations with the M470 allele. J Cyst Fibros 6:15-22.

Cuppens H, Lin W, Jaspers M, et al. (1998) Polyvariant mutant cystic fibrosis transmembrane conductance regulator genes. The polymorphic $(\mathrm{Tg}) \mathrm{m}$ locus explains the partial penetrance of the T5 polymorphism as a disease mutation. J Clin Invest 101:487-496.

de Cid R, Ramos MD, Aparisi L, et al. (2010) Independent contribution of common CFTR variants to chronic pancreatitis. Pancreas 39:209-215.

de Meeus A, Guittard C, Desgeorges M, et al. (1998) Linkage disequilibrium between the M470V variant and the IVS8 polyT alleles of the CFTR gene in CBAVD. J Med Genet 35: 594-596.

DerSimonian R, Laird N (2015) Meta-analysis in clinical trials revisited. Contemp Clin Trials 45:139-145.

Egger M, Davey Smith G, Schneider M, et al. (1997) Bias in meta-analysis detected by a simple, graphical test. BMJ 315 : 629-634.

Fujiki K, Ishiguro H, Ko SB, et al. (2004) Genetic evidence for CFTR dysfunction in Japanese: background for chronic pancreatitis. J Med Genet 41:e55.

Hanck C, Schneider A, Whitcomb DC (2003) Genetic polymorphisms in alcoholic pancreatitis. Best Pract Res Clin Gastroenterol 17:613-623.

International HapMap C (2005) A haplotype map of the human genome. Nature 437:1299-1320.

Issa Y, Bruno MJ, Bakker OJ, et al. (2014) Treatment options for chronic pancreatitis. Nat Rev Gastroenterol Hepatol 11: 556-564.

Keiles S, Kammesheidt A (2006) Identification of CFTR, PRSS1, and SPINK1 mutations in 381 patients with pancreatitis. Pancreas 33:221-227.

Kosova G, Pickrell JK, Kelley JL, et al. (2010) The CFTR Met 470 allele is associated with lower birth rates in fertile men from a population isolate. PLoS Genet 6:e1000974.

Lau J, Ioannidis JP, Schmid CH (1997) Quantitative synthesis in systematic reviews. Ann Intern Med 127:820-826.

Lee JH, Choi JH, Namkung W, et al. (2003) A haplotype-based molecular analysis of CFTR mutations associated with respiratory and pancreatic diseases. Hum Mol Genet 12:23212332.

Moskowitz SM, Chmiel JF, Sternen DL, et al. (2008) Clinical practice and genetic counseling for cystic fibrosis and CFTRrelated disorders. Genet Med 10:851-868.

Pompei F, Ciminelli BM, Bombieri C, et al. (2006) Haplotype block structure study of the CFTR gene. Most variants are associated with the M470 allele in several European populations. Eur J Hum Genet 14:85-93.

Rosendahl J, Landt O, Bernadova J, et al. (2013) CFTR, SPINK1, CTRC and PRSS1 variants in chronic pancreatitis: is the role of mutated CFTR overestimated? Gut 62:582-592.

Steiner B, Rosendahl J, Witt H, et al. (2011) Common CFTR haplotypes and susceptibility to chronic pancreatitis and congenital bilateral absence of the vas deferens. Hum Mutat 32:912-920. 
Stroup DF, Berlin JA, Morton SC, et al. (2000) Meta-analysis of observational studies in epidemiology: a proposal for reporting. Meta-analysis Of Observational Studies in Epidemiology (MOOSE) group. JAMA 283:2008-2012.

Thrower E, Husain S, Gorelick F (2008) Molecular basis for pancreatitis. Curr Opin Gastroenterol 24:580-585.

Tomaiuolo AC, Sofia VM, Surace C, et al. (2015) Relationship between CFTR and CTRC variants and the clinical phenotype in late-onset cystic fibrosis disease with chronic pancreatitis. J Mol Diagn 17:171-178.

Tzetis M, Kaliakatsos M, Fotoulaki M, et al. (2007) Contribution of the CFTR gene, the pancreatic secretory trypsin inhibitor gene (SPINK1) and the cationic trypsinogen gene (PRSS1) to the etiology of recurrent pancreatitis. Clin Genet 71:451-457.
Witt H, Apte MV, Keim V, et al. (2007) Chronic pancreatitis: challenges and advances in pathogenesis, genetics, diagnosis, and therapy. Gastroenterology 132:1557-1573.

Address correspondence to:

Donger Zhou, MD

Department of Hepatobiliary-Pancreatic Surgery

The Second Affiliated Hospital

Zhejiang University School of Medicine

Hangzhou 310009

Zhejiang

China

E-mail: dzhou@zju.edu.cn 